\title{
Effect of seismic wave velocity on the dynamic response of multi-story structures on elastic foundation
}

\author{
Çağlayan Hızal*, Gürsoy Turan \\ Department of Civil Engineering, Izmir Institute of Technology, Urla Izmir, Turkey
}

\section{A R T I C L E I N F O}

\section{Keywords:}

Traveling wave effect

Winkler springs

Soil-structure interaction

Multi support excitation

Displacement loading

Static correction

\begin{abstract}
A B S T R A C T
Traveling wave effects are generally considered with three main cases: (i) Wave passage effect that results with time delay in earthquake motion. (ii) incoherence effect which is defined as loss of coherency in the ground motion due to the reflection and refraction of waves, and (iii) local site effects. For multi-story structures whose supports are close to each other, the incoherence and local site effect may be omitted. In this case, traveling waves result only in a pure time delay in the earthquake motion (wave passage effect). Due to the wave passage effect of vertical and/or horizontal ground motion, the superstructure needs to be analyzed by multi-support excitation. Raft foundations cannot constrain vertical deformations and/or rotations, but they cause a diaphragm effect in the horizontal direction which results in uniform excitation. In this study, the effect of vertical earthquake motions onto multi-story buildings on elastic soil is investigated. Multi support excitation is considered by using displacement loading, which defines the equivalent seismic loads in terms of the ground displacement. According to the performed simulations of the selected structures, it is shown that structural height has a direct influence that results in member force magnifications with slow traveling wave effect. Among these, the ground floor column axial forces are most affected.
\end{abstract}

\section{Introduction}

Dynamic response analysis of structures subjected to multi-support excitation has an increased interest in the earthquake engineering society. Multi support excitation is a well-formed analysis tool that is widely used in earthquake response analysis of structures with large footsteps. Past research showed that seismic waves passing through the ground may cause considerable change in the response of structures, if the supports are far apart. The change in the earthquake wave is not only considered with a time delay, but also should contain the path attenuation, reflection and refraction effects. The local site effect is also prominent for this phenomenon due to the non-homogenous nature of the soil medium. In the literature, many researchers paid attention to local soil effect in multi-support excitation [1-9]. Cui and Gao [10] have investigated the traveling wave effect in long-span cable stayed bridges, and they concluded that long-span cable-stayed bridges not only need to consider traveling wave effect, but also study on refraction, reflection and scattering of the waves in different medium of the underlying soil. Jihong et al. [11] presented a simplified method to estimate multi-support excitation responses. In their study, the multisupport response spectrum was constructed by modification and extension of the existing response spectrum method under uniform excitation. Wang et al. [12] investigated the effect of apparent wave velocity on a long span suspension bridge and determined the design characteristics of long span bridges under traveling earthquake wave effect. Hizal and Turan [13] have investigated the seismic behavior of a cable stayed bridge subjected to different support displacements and concluded that the traveling wave results in a pure time delay in the base shear force response.

In the literature, only a few studies have been observed that deal with traveling wave effect in multi-story frames. Rambabu and Allam [14] investigated the effect of apparent wave velocity in open frame structures with soil structure interaction. Allam [15] investigated the same case by a filtered white noise function to model the local site effects such as reflections and refractions of earthquake waves in soil medium. In these studies, only the horizontal component of the ground motion was considered, and a change is observed in the dynamic response with small time delays in the ground acceleration. This observation may be reliable for the structures whose supports are able to move independently from each other. In many cases, multiple support excitation does not seem feasible to implement in multi-story buildings since they are built on raft foundations in which the supports are not able to move independently. However, the time delay in the vertical ground motion may cause a valuable change in the dynamic response of

\footnotetext{
* Corresponding author.

E-mail address: caglayanhizal@iyte.edu.tr (Ç. Hızal).
} 
the structure since the foundation beam/or slab cannot be assumed infinitely rigid in the vertical direction. In addition, most of the conducted research that is available in the literature considers both incoherence effect and local site conditions in multi-support analysis. These effects, however, may be neglected if the supports of the structure are adequately close to each other. In this case, only the wave passage effect which comes up with time delay in earthquake motion will be prominent in multi-support excitation.

In multi support analysis, pseudo-static displacement effects have an important role on the dynamic responses. For this reason, a transformation is required between the relative and absolute dynamic displacements. The displacement loading which defines the general equation of motion with absolute coordinates appears to be more practical when compared to the conventional acceleration loading. This fact can be explained by the very sensitive behavior of acceleration records to little amplitude changes. For example, a small offset in the acceleration values may result in linearly increasing velocity and quadratic increasing displacements which may easily be overseen. To the contrary, displacement loading is robust with respect to amplitude changes. As a result, when multiple constraints need to be defined by different motions, displacement loading should be the preferred one, which is also validated by other researchers [16-18] and regulated in different software $[19,20]$. In modal analysis of displacement loading, higher modes become dominant because of the pseudo-static effects and it may require nearly all modes to obtain a reliable dynamic response. Tsai [17] summarized the general modal analysis procedure of displacement loading and proposed a static correction method for the consideration of higher mode effects. Hizal and Turan [21] pointed out pseudo static effects in the base shear force response of cable stayed bridges and comprehended on the physical meaning of the static correction method for modal analysis.

A study on traveling wave effect of vertical ground motion in multistory structures with soil structure interaction is not found in the reviewed literature. This study sheds light on the effect of the apparent wave velocity on the dynamic member forces is investigated by using finite element models of three soil-structure interaction systems.

\section{Statement of the problem}

As the earthquake wave velocity is low, a time delay effect can be seen at the support of the structure. To illustrate the problem, one frequency component of an earthquake motion is considered. Fig. 1 shows an example in which a single sine wave type vertical ground

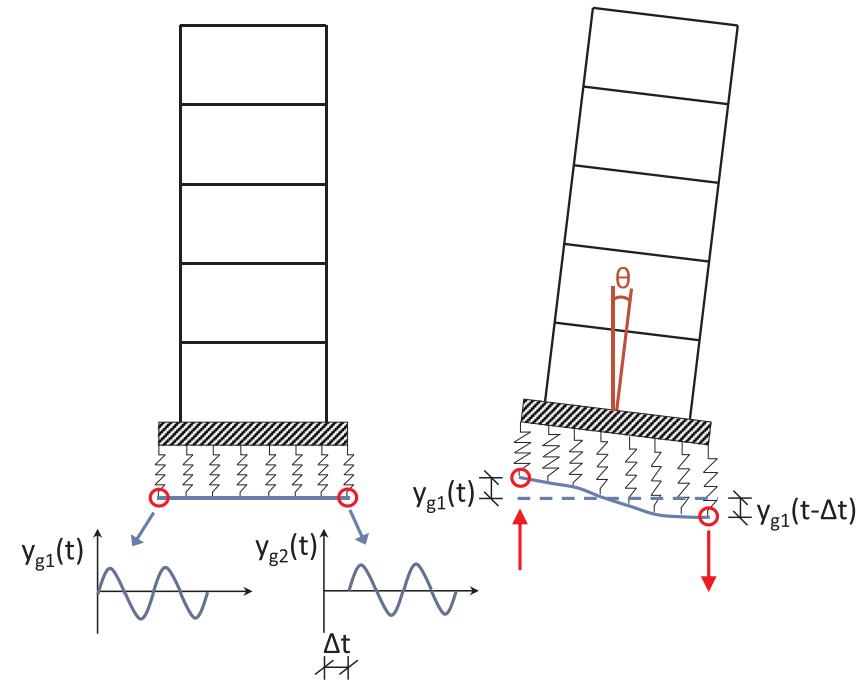

Fig. 1. Schematically representation of the traveling vertical ground motion effect. motion is propagating from left to right. At the extreme case, the left and right ends may deform in opposite directions with maximum amplitude. This situation is possible when the time delay is equal to half of the period of the sinusoidal ground motion, and the wave velocity should be equal to the raft foundation length divided by the time delay.

$V=\frac{L}{\Delta t}=\frac{2 L}{T_{\text {wave }}}$

As an example, for a foundation $\mathrm{L}=30 \mathrm{~m}$, and wave velocity $\mathrm{V}$ $=100 \mathrm{~m} / \mathrm{sec}$, the time delay would be $0.3 \mathrm{~s}$. Then, the extreme case would be seen for a sine wave with a period $\mathrm{T}_{\text {wave }}=0.6 \mathrm{~s}$. The nonextreme cases in which the sine wave period, and/or the wave velocity is different may also cause rotation of the raft foundation.

The above-mentioned response stems from the earthquake wave characteristic and is not related to the inertial overturning effect of the superstructure with soil structure interaction. The two mechanisms, however, may interact leading to a resonance effect.

The ground motion that is investigated in this study is considered to result from surface waves which cause lateral, vertical, and rotational motion. The lateral and vertical motions are implemented in the base of the springs representing the soil medium. The rotational motion of a point on the ground surface (due to Rayleigh waves), however, is not considered. Instead, the modeled springs are placed close to each other so that a relative vertical displacement obtained from earthquake records may be considered as a rotational motion of the ground. The differential vertical ground displacements occur due to the ground motion traveling underneath the foundation. The ground motion at a point is a composition of $\mathrm{P}, \mathrm{S}$, and surface waves (e.g. Rayleigh wave) which according to Kramer (1996) have different propagation speeds. Fig. 2 shows the wave speed ratio to shear wave velocity, $V_{s}$, with respect to Poisson's ratio, $\mu$, of the soil medium. The shear wave velocity is calculated as

$V_{s}=\sqrt{\frac{G}{\rho}}$

where $G=$ shear modulus and $\rho=$ unit mass of soil. For $\mu=0$, the speed of Rayleigh waves is $20 \%$ less than the shear wave velocity and they are equal when $\mu=0.5$, which corresponds to clay type soil. The $P$ wave velocity exponentially increases as $\mu$ increases. The expected ground motion due to $\mathrm{P}$ waves, however, are not expected to be of important magnitude when compared to the $S$ and surface wave effects. For this study, a differentiation of the Rayleigh and shear waves is not performed and their propagating velocity is assumed to be identical.

In the horizontal direction, the ground will experience different motion underneath the foundation. Raft foundations, however, are rigid and do not elongate. Therefore, a uniform lateral motion is expected in

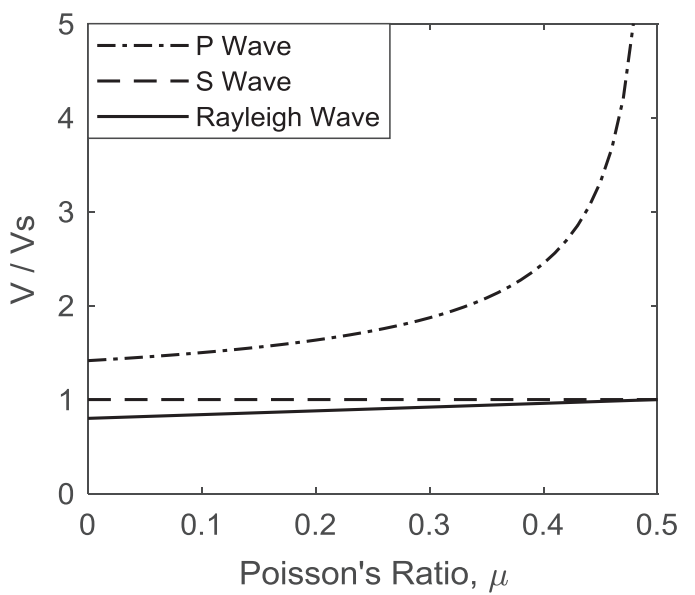

Fig. 2. Variations of seismic wave propagation speeds versus Poisson's ratio [22]. 


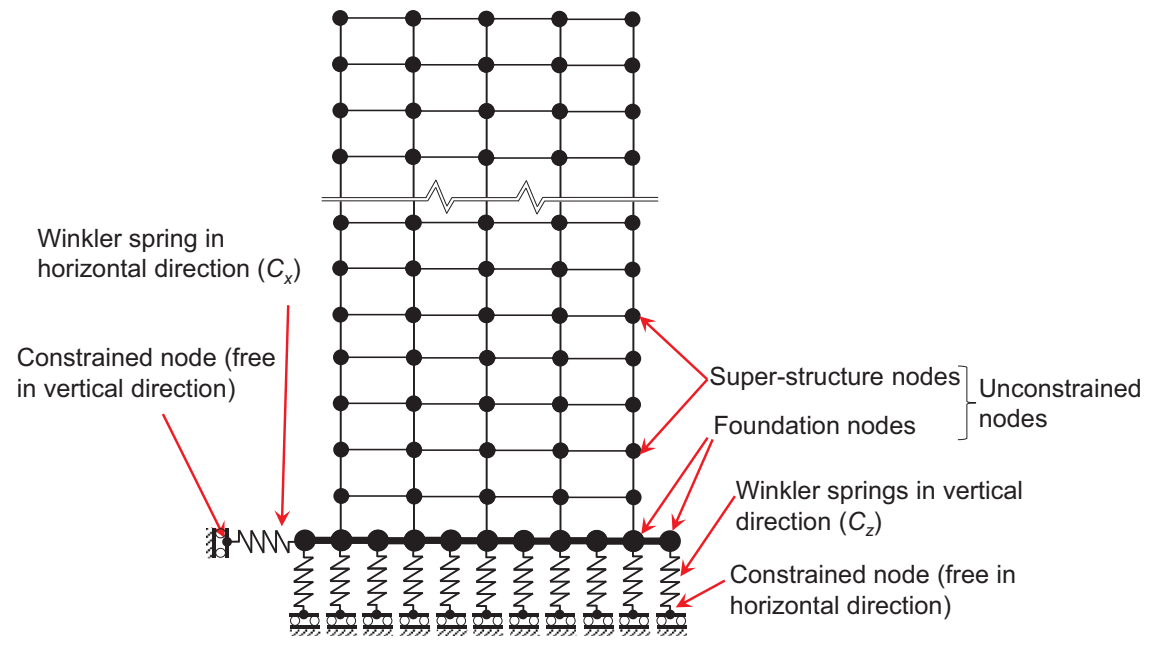

Fig. 3. Schematically representation of the presented mathematical model.

the horizontal direction. As a result, a single spring model appears to be sufficient in modeling the lateral soil stiffness.

\section{Mathematical formulation}

The mathematical model of a soil structure interaction (SSI) system is shown in Fig. 3. The soil stiffness is represented by elastic springs (Winkler springs). The mathematical model is constructed according to the following assumptions; (i) equivalent soil springs are assumed to be linear elastic, (ii) material and geometrical nonlinearities are neglected, (iii) damping of soil is neglected, (iv) mass of Winkler springs are neglected.

In the finite element formulation of the structure with SSI, the stiffness, mass and damping matrices contain the structural information of both superstructure and foundation components. The equation of motion of the system presented in Fig. 3 is written as

$\left[\begin{array}{cc}M & 0 \\ 0 & 0\end{array}\right]\left\{\begin{array}{c}\ddot{y}^{t}(t) \\ \ddot{y}_{g}(t)\end{array}\right\}+\left[\begin{array}{cc}C & 0 \\ 0 & 0\end{array}\right]\left\{\begin{array}{c}\dot{y}^{t}(t) \\ \dot{y}_{g}(t)\end{array}\right\}+\left[\begin{array}{cc}K & K_{g} \\ K_{g}^{T} & K_{g g}\end{array}\right]\left\{\begin{array}{c}y^{t}(t) \\ y_{g}(t)\end{array}\right\}=\left\{\begin{array}{c}0 \\ F_{g}(t)\end{array}\right\}$

in which $M, C$ and $K$ are the mass, stiffness and damping matrices of the unconstrained (superstructure) DOF, $K_{g g}$ is the stiffness matrix of the constrained (ground) DOF, and $K_{g}$ denotes the coupled stiffness due to the Winkler springs. In addition, $y^{t}(t)$ and $y_{g}(t)$ are the absolute displacement vector of the unconstrained and constrained DOF. At the right-hand side of Eq. (3), $F_{g}(t)$ denotes the equivalent external dynamic force acting on the constrained DOF. This force is considered to be the action force of the ground, that activates the constrained DOF.

The first and second row of Eq. (3) gives the equation of motion of the unconstrained and constrained DOF, respectively.

$M \ddot{y}^{t}(t)+C \dot{y}^{t}(t)+K y^{t}(t)=-K_{g} y_{g}(t)$

$K_{g}^{T} y_{g}(t)+K_{g g} y_{g}(t)=F_{g}(t)$

The equivalent elastic force vector acting on the unconstrained DOF is obtained by

$f_{s}(t)=K y^{t}(t)+K_{g} y_{g}(t)$

Which is equivalent to

$f_{s}(t)=K y(t)$

in which $y(t)$ denotes the relative displacement vector of the unconstrained DOF with respect to the constrained DOF. Thus, the following relation can be constructed between the displacement of constrained and unconstrained DOF by equating Eqs. (6) and (7).

$y^{t}(t)=y(t)+\ell y_{g}(t)$

Here $\ell$ denotes the $N \times N_{g}$ size influence matrix which defines a relation between pseudo-static displacements of the unconstrained DOF and constrained displacements and can be evaluated as [23]

$\ell=-K^{-1} K_{g}$

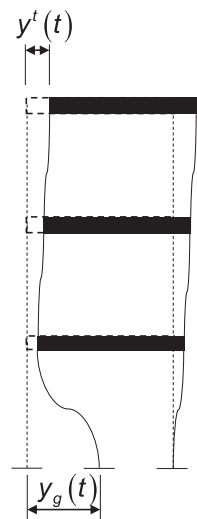

Absolute Displacement Case
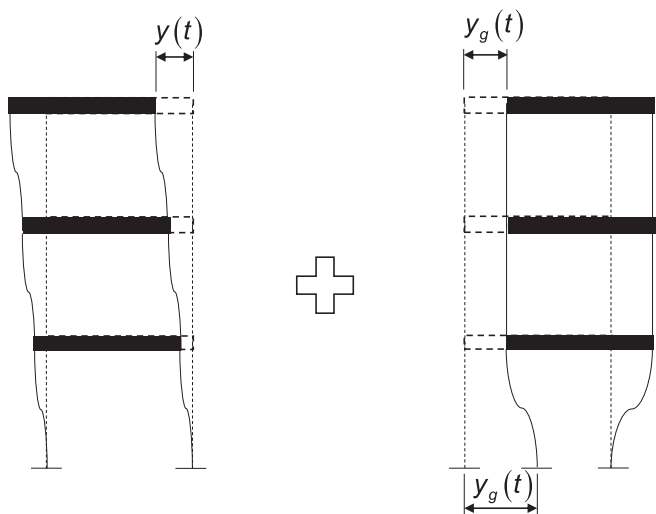

Relative Displacement Case

Pseudo-static Displacement Case

Fig. 4. Illustration of the absolute, relative and pseudo-static displacement cases. 

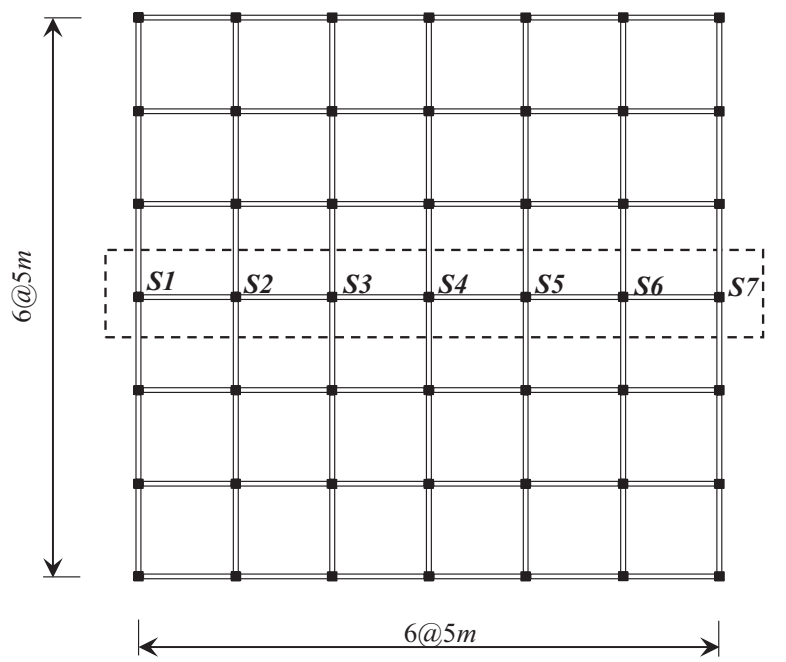

Story height $=300 \mathrm{~cm}$

Columns $=75 \times 75 \mathrm{~cm}$

Beams $=30 \times 70 \mathrm{~cm}$

Slab thickness $=15 \mathrm{~cm}$

$E_{C}=30 \mathrm{Mpa}$

Modal damping ratio, $\xi=5 \%$

Fig. 5. Plan view of the modeled reinforced concrete structures.

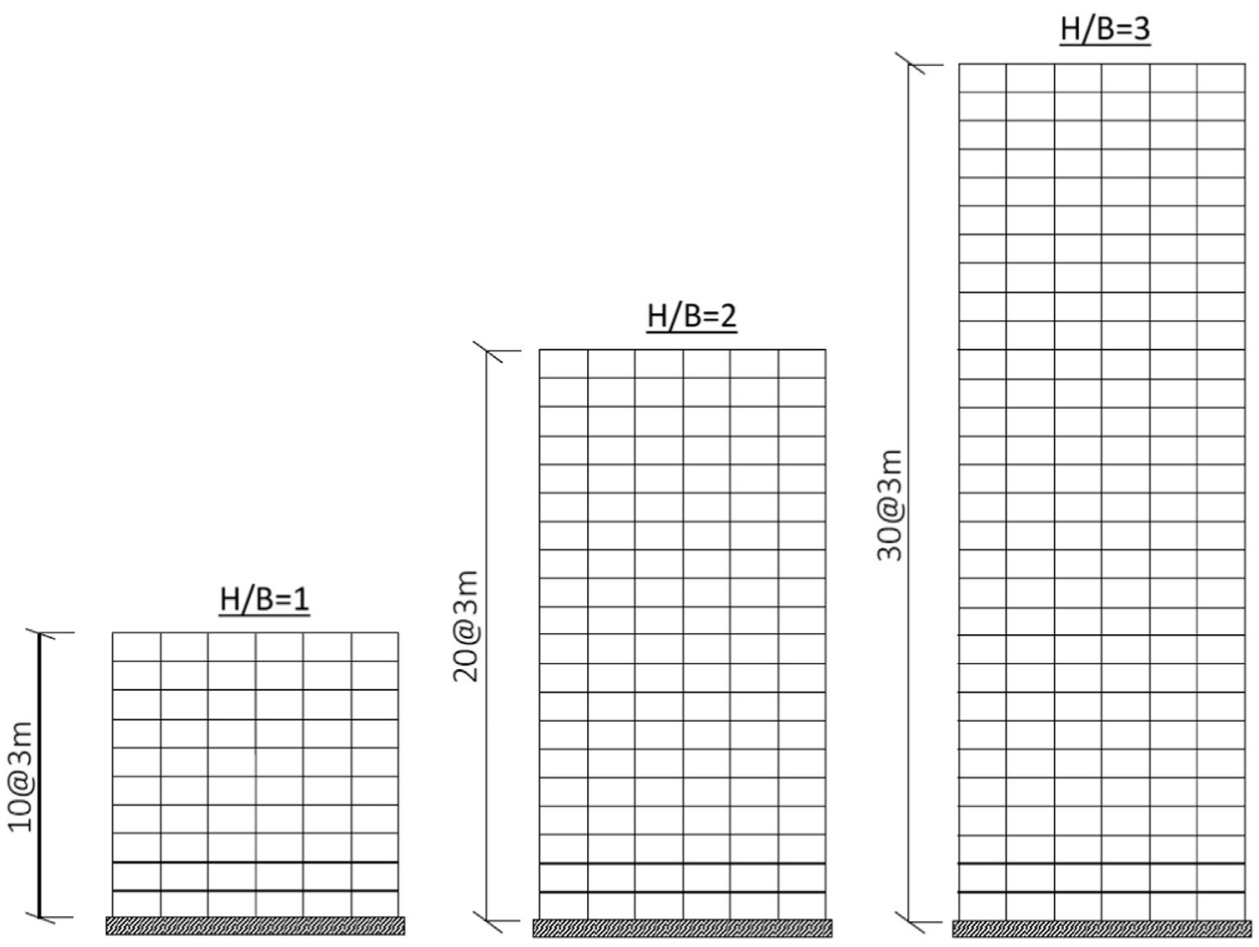

Fig. 6. Elevation view of the modeled reinforced concrete structures.

Table 1

Calculated shear modulus for various shear wave velocities.

\begin{tabular}{lllll}
\hline Vs (m/sec) & 50 & 100 & 500 & 1000 \\
\hline G (MPa) & 0.50 & 20 & 510 & 2039 \\
\hline
\end{tabular}

Accordingly, the second term of Eq. (8) denotes the pseudo static structural displacements. In Fig. 4 the schematic representation of the decomposition of absolute displacements is presented.

\section{Modal analysis procedure}

In the modal analysis, the displacement vector of the unconstrained DOF can be written as
$y^{t}(t)=\sum_{i=1}^{N} \phi_{i} q_{i}(t)$

where $\phi_{i}$ and $q_{i}(t)$ are the modal shape vector and normal coordinate function of the $i$ th mode. The uncoupled equation of motion of the $i$ th mode can be obtained by substituting Eq. (10) into Eq. (4) and left multiplying each side by $\phi_{i}{ }^{T}$ results in the following;

$M_{i} \ddot{q}_{i}(t)+C_{i} \dot{q}_{i}(t)+K_{i} q_{i}(t)=-\phi_{i}^{T} K_{g} y_{g}(t)$

where $M_{i}, C_{i}$ and $K_{i}$ denote the generalized mass, damping and stiffness matrices of the $i$ th mode, respectively.

$M_{i}=\phi_{i}^{T} M \phi_{i}$
$C_{i}=\phi_{i}^{T} C \phi_{i}$
$K_{i}=\phi_{i}^{T} K \phi_{i}$

The equivalent load vector given at the right-hand side of Eq. (11) is 
a-)Horizontal Direction
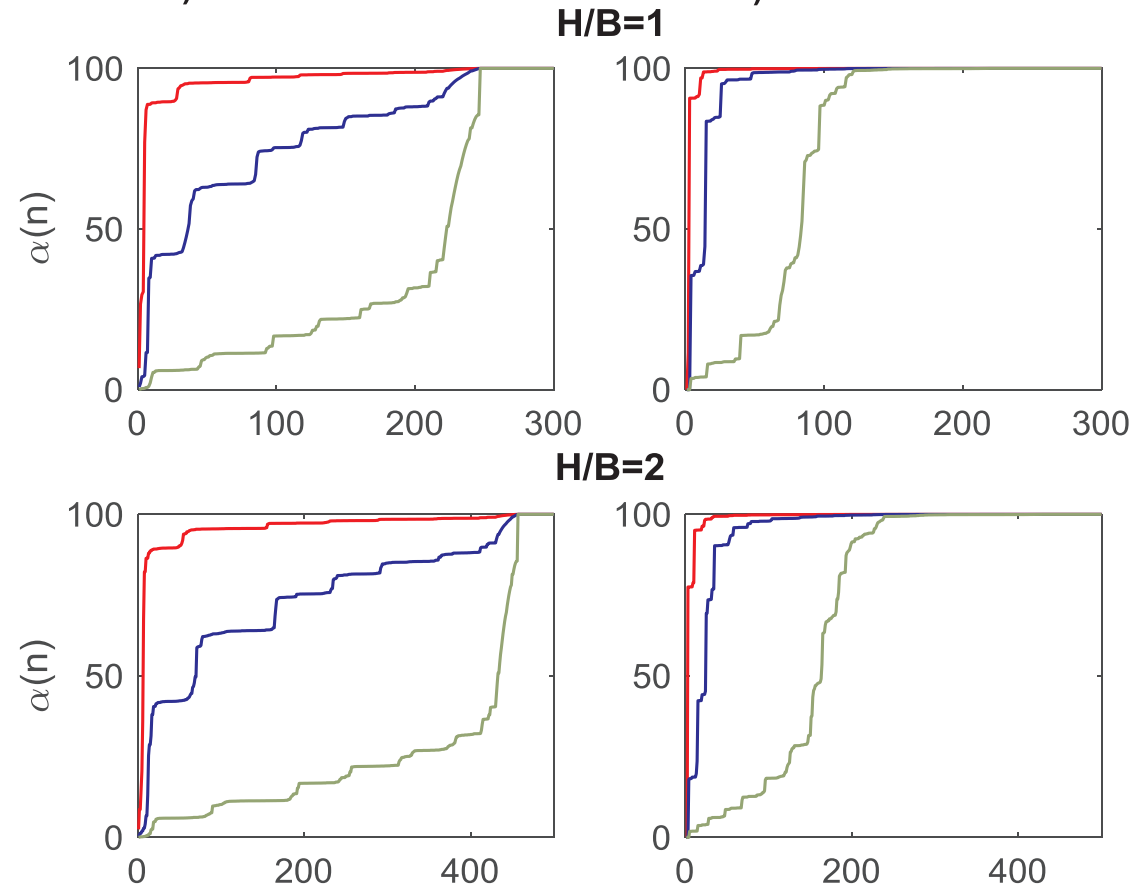

$\mathrm{H} / \mathrm{B}=3$

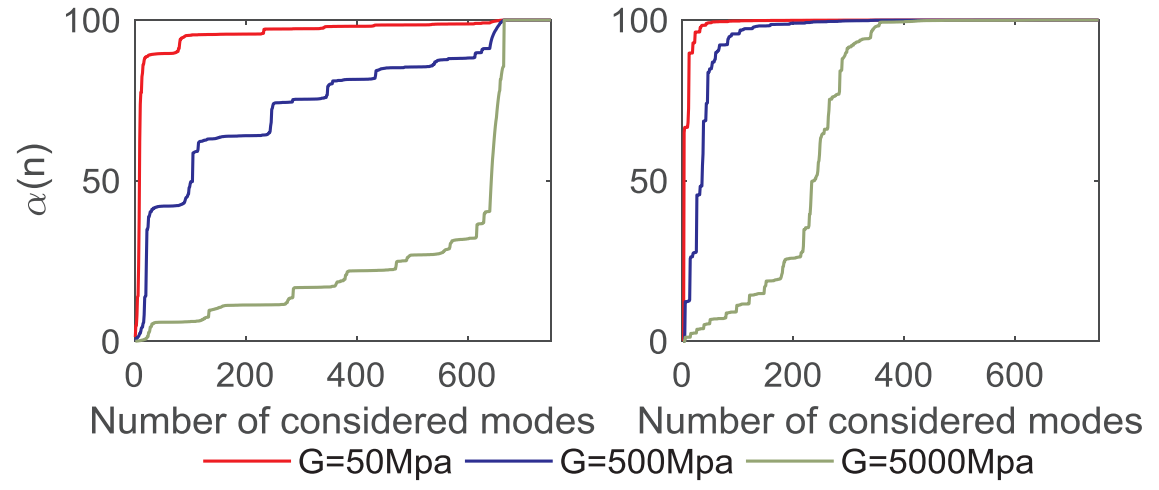

Fig. 7. Variations in $\alpha(n)$ for (a) lateral (horizontal) and (b) vertical ground motions.

proportional to the ground displacement and it is referred to as displacement loading in the literature. If both sides of Eq. (11) is divided by $M_{i}$, the uncoupled equation of motion will turn into the following form:

$\ddot{q}_{i}(t)+2 \xi_{i} \omega_{i} \dot{q}_{i}(t)+\omega_{i}^{2} q_{i}(t)=\Gamma_{i}^{K} y_{g}(t)$

in which $\xi_{i}, \omega_{i}$ and $\Gamma_{i}^{k}$ indicate the damping ratio, natural angular frequency and modal participation factor of the $i^{\text {th }}$ mode.

$\Gamma_{i}^{K}=\frac{-\phi_{i}^{T} K_{g}}{\phi_{i}^{T} M \phi_{i}}$

Note that the modal participation factor given in Eq. (14) is associated with constrained DOF displacements. If Eq. (9) is substituted into the right-hand side of Eq. (14), the following equation will be obtained.

$\Gamma_{i}^{K}=\frac{\phi_{i}^{T} K \ell}{\phi_{i}^{T} M \phi_{i}}$

Eq. (15) turns into the following form when ${\phi_{i}}^{T} K_{u u}$ is substituted by $-\phi_{i}^{T} \omega_{i}^{2} M$.

$\Gamma_{i}^{K}=\Gamma_{i}^{M} \omega_{i}^{2}$

$\Gamma_{i}^{M}$ denotes the modal participating factor obtained for acceleration loading (it is a mass proportional parameter, whereas $\Gamma_{i}^{K}$ is a stiffness proportional parameter.).

$\Gamma_{i}^{M}=-\frac{\phi_{i}^{T} M \ell}{\phi_{i}^{T} M \phi_{i}}$

\section{Static correction method}

It is known from the literature that the displacement loading excites higher modes of vibration $[17,18,21]$. The reason of this fact can be deducted by considering the physical meaning of Eq. (16). In the conventional acceleration loading in which the equation of motion is constructed with relative displacements, the modal participation decreases in the higher modes. This case also causes a decrease in the higher mode responses. For displacement loading, however, the modal participation factor is amplified by $-\omega_{i}^{2}$. Consequently, the response of the higher modes may dominate the total response. In order to overcome this problem, a static correction procedure can be applied. The higher modal responses are taken into account by using the pseudo static component of the absolute displacements.

The modal expansion of the spatial distribution of constrained displacements can be written as follows [21]. 

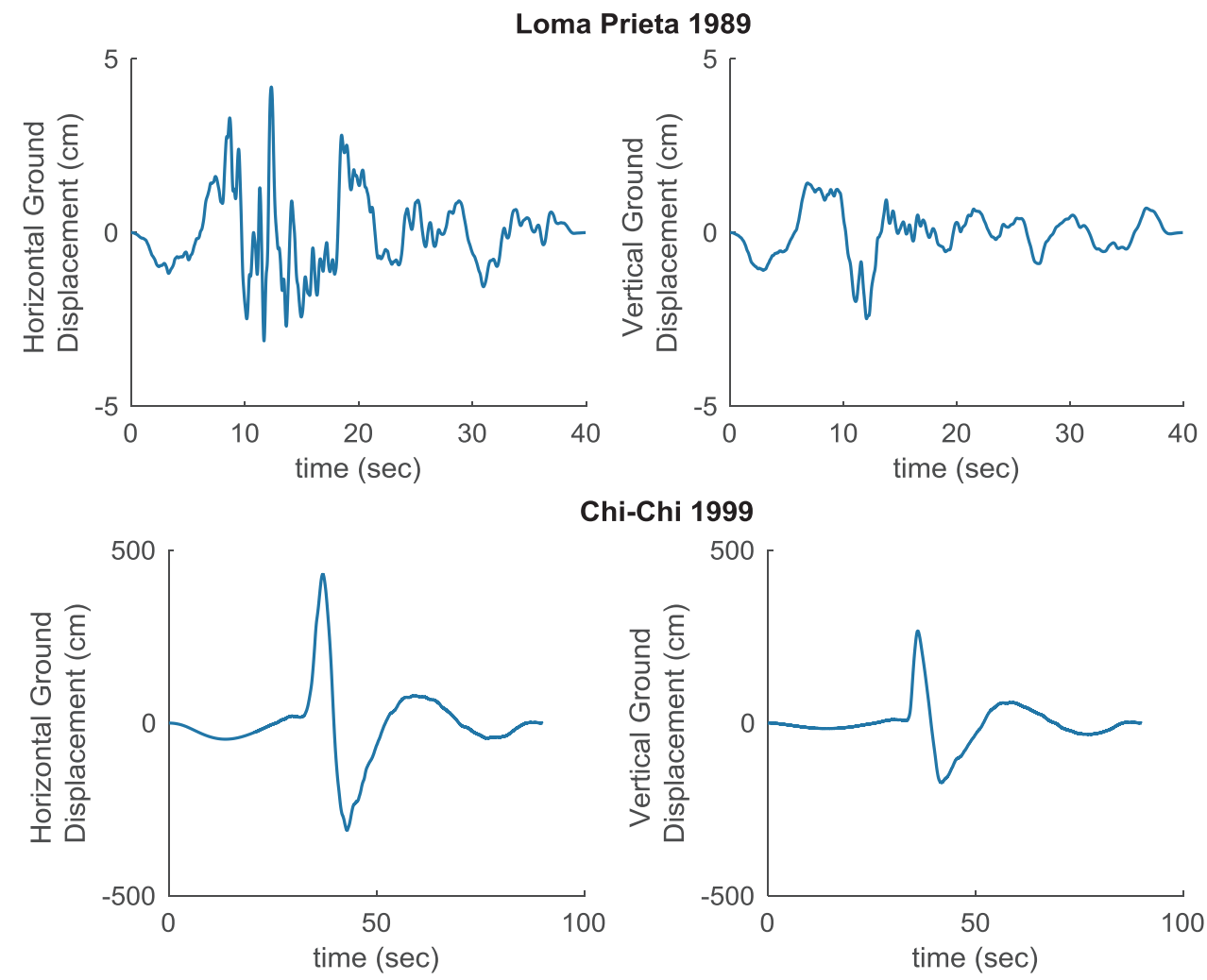

Chi-Chi 1999

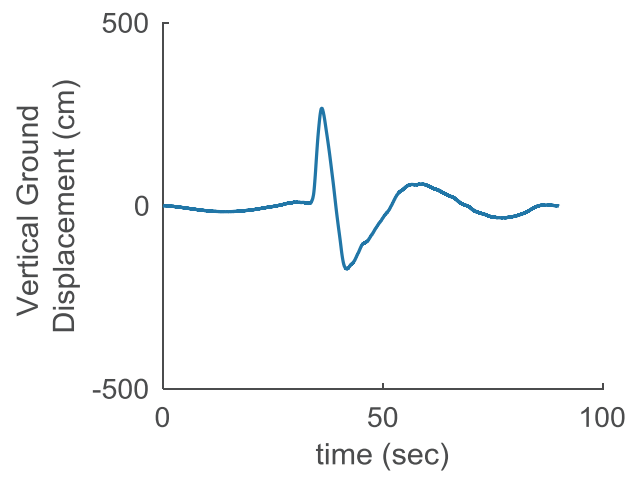

Fig. 8. Vertical and horizontal component of selected ground motions.

$K_{g}=-\sum_{i=1}^{N} M \phi_{i} \Gamma_{i}^{K}$

Substituting $M \phi_{i}$ with $-\omega_{i}^{-2} K \phi_{i}$ and arranging the results yields

$\ell=\sum_{i=1}^{N} \phi_{i} \Gamma_{i}^{K} \omega_{i}^{-2}$

Thus, the higher mode response is represented by its pseudo-static component as

$y_{s c}(t)=\left[\ell-\sum_{i=1}^{n} \phi_{i} \Gamma_{i}^{K} \omega_{i}^{-2}\right] y_{g}(t)$

where $n$ denotes the number of considered modes. Finally, the corrected absolute displacement response is obtained as

$y^{t}(t)=\sum_{i=1}^{n} \phi_{i} q_{i}(t)+y_{s c}(t)$

\section{Modal contribution of pseudo-static effects}

In the conventional acceleration loading approach, pseudo-static effects do not need to be considered separately since relative displacements already contain these effects. In displacement loading, however, the pseudo-static effects become dominant. In the previous section, it is stated that higher modal response effects are caused by pseudo-static components. The physical meaning of this phenomenon arises from the modal decomposition of the absolute, relative and pseudo static displacements. The modal decomposition of the relative displacements is given by

$y(t)=\sum_{i=1}^{N} \phi_{i} \rho_{i}(t)$

where $\rho_{i}(t)$ denotes the normal coordinate of the relative displacements.
In addition, it is seen that the modal shape vector is the same for both absolute and relative displacements. Therefore, the only difference between the relative and absolute displacements originates from the difference in normal coordinates of absolute and relative displacements. By substituting Eqs. (20) and (22) into Eq. (21) one can obtain the following result.

$\sum_{i=1}^{n} \phi_{i} \rho_{i}(t)+e y_{g}(t)=\sum_{i=1}^{n} \phi_{i} q_{i}(t)+\left[R-\sum_{i=1}^{n} \phi_{i} \Gamma_{i}^{K} \omega_{i}^{-2}\right] y_{g}(t)$

After some manipulations, the difference between normal coordinates is obtained as

$q_{i}(t)-\rho_{i}(t)=\Gamma_{i}^{M} y_{g}(t)$

$=\frac{-\phi_{i}^{T} K_{g} y_{g}(t)}{\omega_{i}^{2} \phi_{i}^{T} M \phi_{i}}$

The right-hand side of Eq. (24) denotes the normal coordinate of the pseudo-static displacements for the ith mode. It can be seen that the modal (or normal) coordinate of pseudo static displacements can be obtained by the ratio of the generalized modal load and the modal stiffness. Thus, the normal coordinate of the absolute displacements can be represented as the summation of the normal coordinates of the relative and the pseudo-static displacements.

$q_{i}(t)=\rho_{i}(t)+\delta_{i}(t)$

where $\delta_{i}(t)=\Gamma_{i}^{M} y_{g}(t)$. Pre-multiplying Eq. (24) by $\phi_{i}$ results in the displacement response due to the $i^{\text {th }}$ mode.

$\phi_{i} q_{i}(t)=\underbrace{\varphi_{i} \rho_{i}(t)}_{\begin{array}{c}\text { relative } \\ \text { displacement }\end{array}}+\underbrace{\varphi_{i} \delta_{i}(t)}_{\begin{array}{c}\text { pseudo-static } \\ \text { displacement }\end{array}}$

Here, the second term represents the modal pseudo static contribution to the structural displacements. To show the importance of higher modes in the pseudo static displacements, the modal potential energy due to the pseudo static displacements can be defined as; 

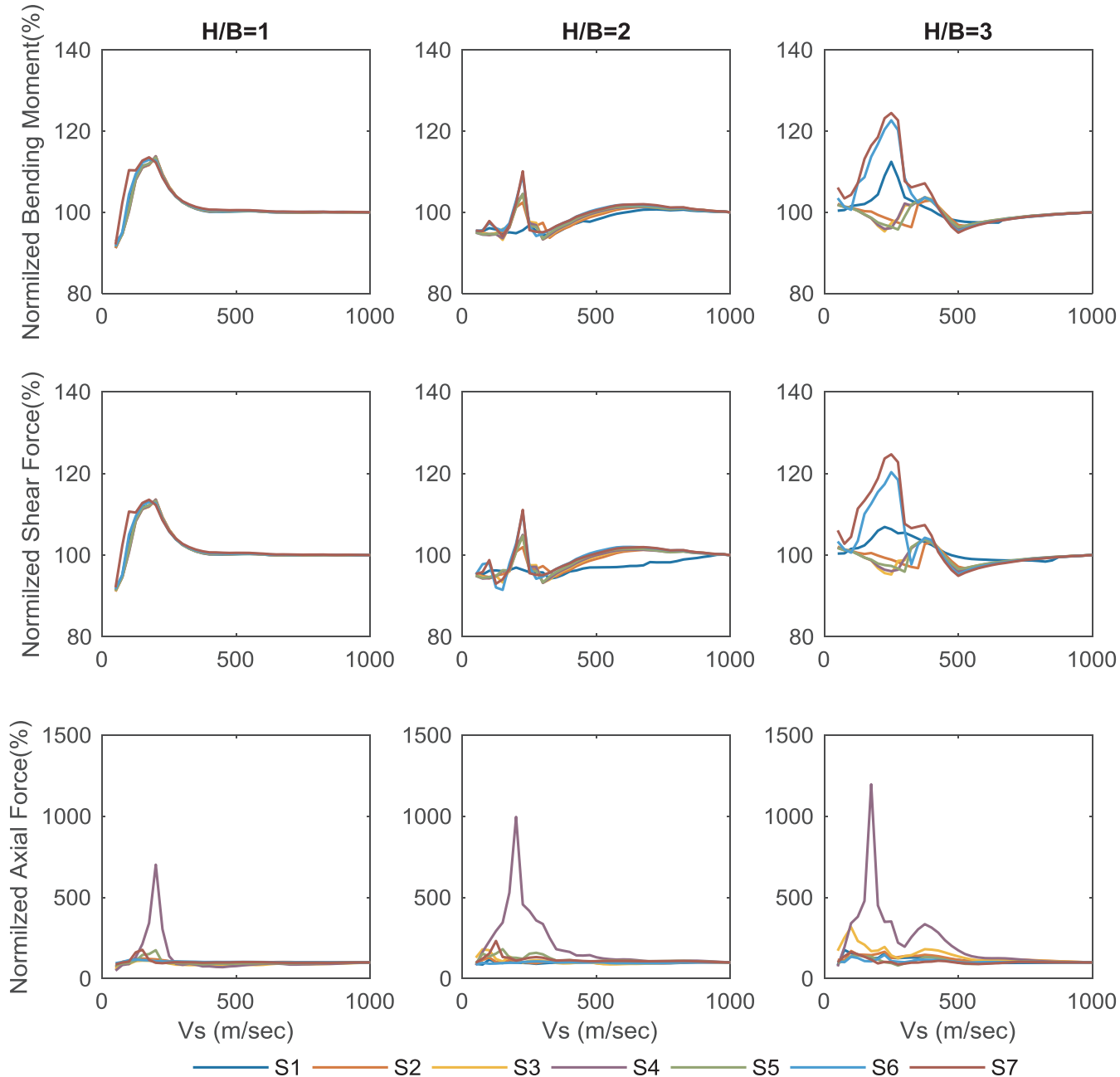

Fig. 9. Variations in the normalized forces acting on the first story columns versus apparent wave velocity for the Loma Prieta 1989 Earthquake.

$W_{i}(t)=\frac{1}{2} \phi_{i}^{T} K \phi_{i} \delta_{i}(t)^{2}$

Then, an energy based estimator can be formulated for comparison purposes. The chosen estimator is shown in Eq.(28) which indicates the percentage of modal potential energies that is accumulated by the first $n$ modes as a ratio to the total potential energy due to the pseudo static displacements. Further, by considering the maximum value, the time variable is removed.

$\alpha(n)=\max \left[\frac{\sum_{i=1}^{n} W_{i}(t)}{\left[\ell y_{\mathrm{g}}(t)\right]^{T} K\left[\ell y_{\mathrm{g}}(t)\right]}\right]$

\section{Numerical analysis}

Three buildings on $1 \mathrm{~m}$ thick raft foundations are considered in this investigation. The buildings have the same base dimensions, and their heights are $30 \mathrm{~m}, 60 \mathrm{~m}$, and $90 \mathrm{~m}$. The plan and elevation views are presented in Figs. 5 and 6, respectively. In the analysis, a two-dimensional finite element model of a selected strip is constructed. The considered strip of the raft foundation is modeled as frame elements with a width of $5 \mathrm{~m}$ and length of $1.0 \mathrm{~m}$.

The considered soil medium is assumed to have a unit mass of $\rho=2038.74 \mathrm{~kg} / \mathrm{m}^{3}$, and Poisson's ratio of 0.20 . For a given shear wave velocity, the shear modulus of soil is evaluated by making use of Eq. (2). Table 1 shows representative shear wave velocities versus the calculated shear modulus values.
The elastic soil stiffness is calculated as follows,

$$
\begin{aligned}
& k_{z}=\frac{G B}{1-\mu}\left[3.1\left(\frac{L}{B}\right)^{0.75}+1.6\right] \\
& k_{x}=\frac{G B}{2-\mu}\left[6.8\left(\frac{L}{B}\right)^{0.65}+0.8\left(\frac{L}{B}\right)+1.6\right], \quad L \geq B
\end{aligned}
$$

where $k_{z}$ and $k_{x}$ represent single elastic spring constants of the subgrade stiffness in the vertical and horizontal directions, $L$ and $B$ are the dimensions of the raft foundation. The given formulas are presented by Pais and Kausel [24] for structures on rectangular rigid foundations. The distributed equivalent spring stiffness which are modeled for the considered strip in the vertical and horizontal directions are calculated as $C_{z}$ and $C_{x}$, respectively. Since all constraint DOFs are assumed to be subjected to the same horizontal ground motion, the horizontal stiffness of the soil is represented by a single spring. Therefore, $C_{x}$ is taken as $\frac{1}{6}$ $k_{x}$. In the vertical direction a vertical elastic spring is assigned for each foundation node so that the resultant soil stiffness in the vertical direction is obtained as [25],

$C_{z}=\frac{k_{z}}{4 B L} \Delta A$

where $\Delta A=5 \times 1.0 \mathrm{~m}$.

The numerical analysis consists of two parts. First part presents a short discussion on the modal variation of pseudo static effects. In the second part, the effect of apparent wave velocity on design forces (bending moment, shear and axial forces) is investigated in detail. 

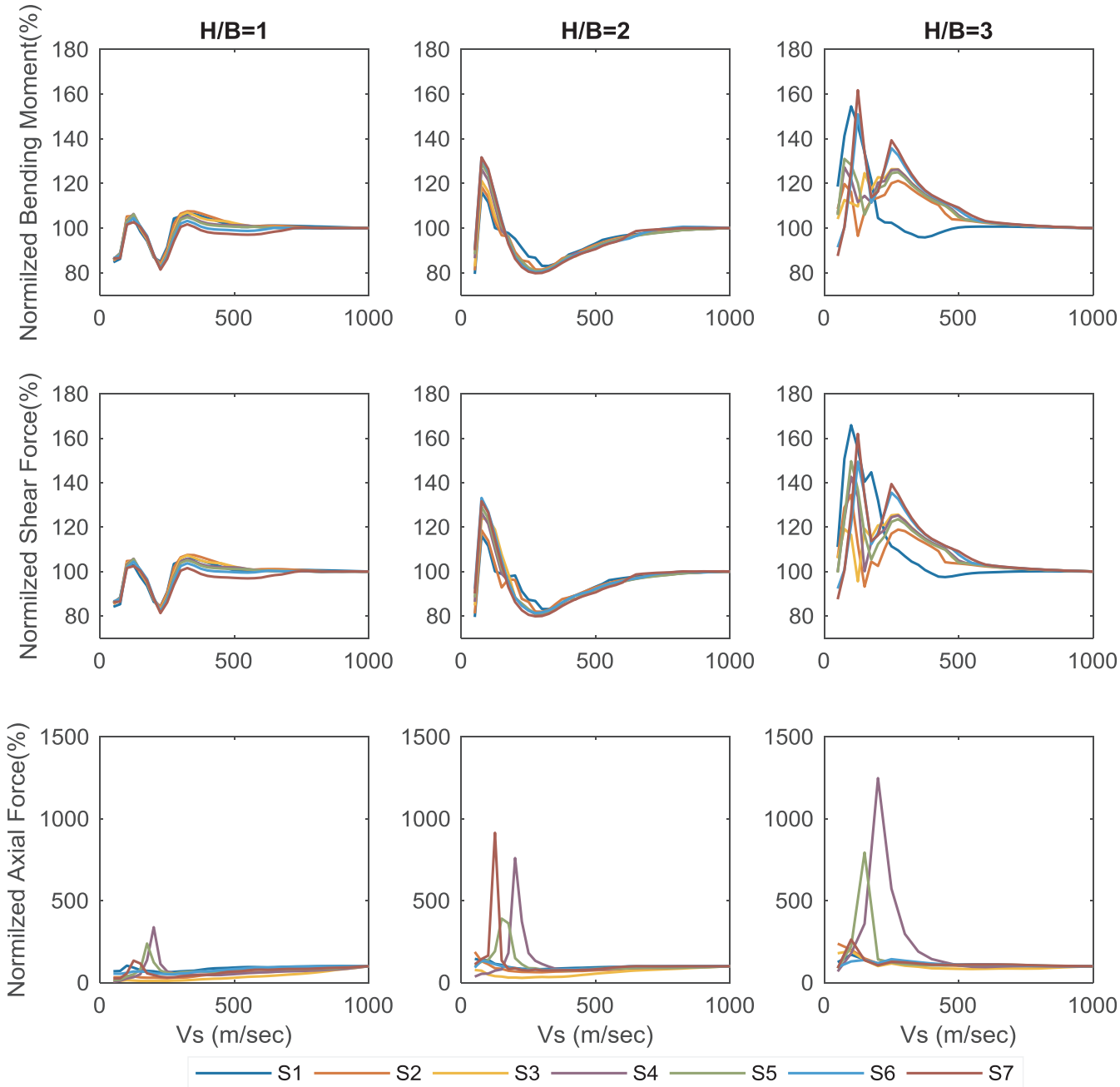

Fig. 10. Variations in the normalized forces acting on the first story columns versus apparent wave velocity for the Chi-Chi 1999 Earthquake.

\subsection{Investigation of the modal contribution of pseudo-static effects}

The ground motion, $y_{g}(t)$, appears in Eq. (28) in quadratic form both in the numerator and denominator. Therefore, the variation of $\alpha(n)$ for the numerical models can be evaluated by assuming unit ground motion, both in the horizontal and vertical direction, separately. Fig. 7 presents the variation of $\alpha(n)$ for horizontal and vertical ground motion with respect to the considered number of modes.

The variation of $\alpha(n)$ with respect to $n$ is obtained for $\mathrm{G}=50,500$, $5000 \mathrm{MPa}$. For the soft soil types, it can be seen that the pseudo-static components are not effective in the larger modes. For this case, static correction may not be necessary since $100 \%$ energy participation is obtained with a few number of modes. However, as the foundation stiffness increases, pseudo-static effects are more and more dominated by higher modes. This behavior is especially pronounced in the case of horizontal ground motion, and the effect is delayed for more stiffer soils when vertical ground motion is considered. As the H/B ratio changes, the variation of $\alpha(n)$ with respect to the considered mode number appears to be unaffected.

\subsection{Effect of apparent wave velocity on design forces}

The aim of this section is to show that the apparent wave velocity may cause a change in the member design forces. For this purpose, two different earthquake records are considered. These are the horizontal and vertical displacement components of the Loma Prieta 1989, and Chi-Chi 1999 earthquakes (see Fig. 8). The earthquake wave is considered to enter the structure (Fig. 5) from the left. According to the range of wave speeds $(50-1000 \mathrm{~m} / \mathrm{sec})$ that are considered in the analysis, a corresponding time delay is added to the vertical ground DOFs. The results are normalized with respect to the uniform loading (without time delay) of the same record.

The Chi-Chi 1999 earthquake shows the characteristics of a near fault ground motion. It is a pulse-like motion with a large period of almost $20 \mathrm{~s}$ and results in very large displacements. The Loma Prieta 1989 earthquake shows the characteristics of both near and far fault type ground motions. Its motion consists of long and short period waves. When considering a small time delay, the maximum difference between vertical ground displacements of the left and right end of the foundation is about $63 \mathrm{~cm}$ for the Chi-Chi 1999 earthquake, and $1.5 \mathrm{~cm}$ for the Loma Prieta 1989 earthquake.

Fig. 9 presents the simulation results for the maximum normalized member forces versus shear wave velocity obtained for the Loma Prieta 1989 earthquake. The variation of the member forces is considered to stem from time-delay effect and soil-structure interaction. The member forces obtained with time delay effect are normalized by the member forces due to uniform excitation. Therefore, the presented variation in Fig. 9 exhibits the time delay effect. It can be seen that, the first story column bending moments can be $13 \%, 10 \%, 25 \%$ larger for $\mathrm{H} / \mathrm{B}=1,2$, 3 , respectively. The same trend can be observed for the shear forces. The axial forces, however, show a much larger increase which is in the order of $700 \%, 1000 \%, 1200 \%$, again for $\mathrm{H} / \mathrm{B}=1,2,3$, respectively, for the central column. For the edge columns, this ratio is seen to be as high as $230 \%$. 


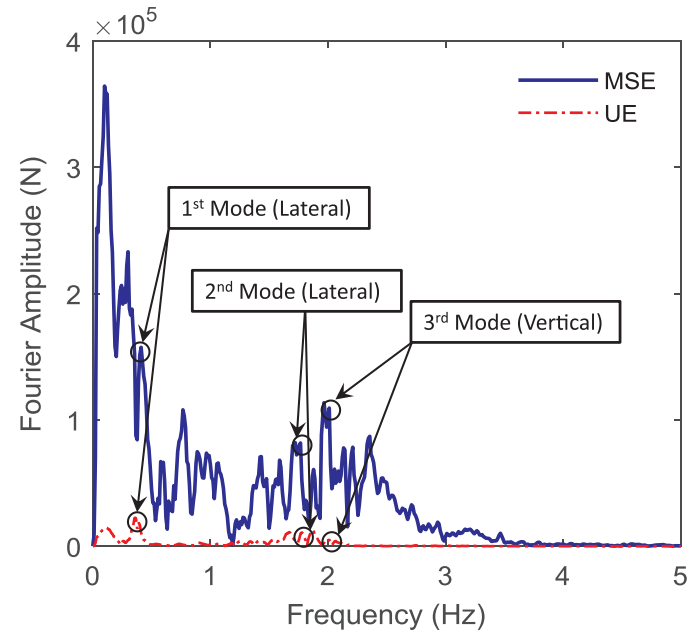

Fig. 11. Fourier amplitudes of the axial force of column S4 for Chi 1999 earthquake in case of multiple (MSE) and uniform (UE) support excitations.

Fig. 10 shows the normalized element force variations with respect to Chi-Chi 1999 earthquake. The first story normalized bending moments have a range of $80 \%-105 \%, 80 \%-130 \%, 90 \%-160 \%$ for $\mathrm{H} /$ $\mathrm{B}=1,2$, and 3 , respectively. A similar variation can be detected from the shear force response versus shear wave velocity. The axial force change is again very high for the center column and its maximum values are $350 \%, 750 \%, 1200 \%$ for $\mathrm{H} / \mathrm{B}=1,2$, and 3, respectively. Different from the Loma Prieta 1989 earthquake response, here, column S7 which is an end column undergoes the largest axial force amplification. Its axial force reaches to $900 \%$ of its uniform excitation value, when $\mathrm{H} / \mathrm{B}=2.0$.

The large axial force values in the mid-columns is attributed to the fact that the time delay may activate vertical structural modes. In fact, $\mathrm{S} 7, \mathrm{~S} 5$, and S4 undergo large axial forces for different wave velocities of $125 \mathrm{~m} / \mathrm{sec}, 150 \mathrm{~m} / \mathrm{sec}$, and $200 \mathrm{~m} / \mathrm{sec}$, respectively. Among these, columns S4 and S5 are located close to the mid-span, and the large values can be considered to stem from the fact that their uniform excitation values are low. This is achievable from a rocking motion in which the end columns will experience larger axial forces than the central columns. Due to the traveling wave effect, the increase in vertical modal response appears to be largest in central columns.

Fig. 11 presents the Fast Fourier Transform (FFT) amplitudes of the axial force response. The wave speed that is used to evaluate the SSI effect is picked from Fig. 10 at which the axial force of S4 is at its maximum for $\mathrm{H} / \mathrm{B}=3(\mathrm{Vs}=200 \mathrm{~m} / \mathrm{sec})$. As a result of time delay, a pronounced increase in the response magnitudes is visible.

For the uniform excitation, the response is mainly composed of the first mode (first combined lateral and rocking mode), the second mode (second combined lateral and rocking mode), third mode (vertical mode) and pseudo static effects at around $0.15 \mathrm{~Hz}$. For the multi-support excitation, however, the pseudo static effects are much more pronounced than the uniform excitation. The increase in the responses is twenty-fold for the vertical mode, ten-fold for the first lateral mode, five-fold for the second lateral mode and pseudo static effects show a more prominent increase when compared to the prior two responses.

Fig. 12 shows the variations in the vertical drift ratio of the raft foundation for multiple and uniform excitations. The vertical drift ratio is equal to the maximum difference between vertical end displacements of the raft foundation divided by foundation length. A first view from the drift ratios indicates a resonance action for $\mathrm{H} / \mathrm{B}=1$, and 3 at low shear wave velocities and the amplitude rise seven to ten times.

For the Loma Prieta 1989 earthquake, time delay has a limited effect on the vertical drift ratios for $\mathrm{H} / \mathrm{B}=1$, and 2 . This small effect appears to be the cause in a variation of $10 \%$ in the bending moment and shear forces. For $\mathrm{H} / \mathrm{B}=3$, however, the maximum difference is in the range of $35 \%$ which is reflected to the variation of the bending moment and shear forces as shown in Fig. 9.

For the Chi-Chi 1999 earthquake and $\mathrm{H} / \mathrm{B}=1$, the maximum drift ratio is slightly larger for the uniform excitation. The difference is in the range of $-10 \%$ which is represented as a reduction in bending moment and shear forces in Fig. 10. For $\mathrm{H} / \mathrm{B}=2$, first of all the drift is quite small. In addition, the variation of drift that is associated due to the time delay is seen to be about $10 \%$. The resulting shear forces and

\section{a-) Loma Prieta 1989}
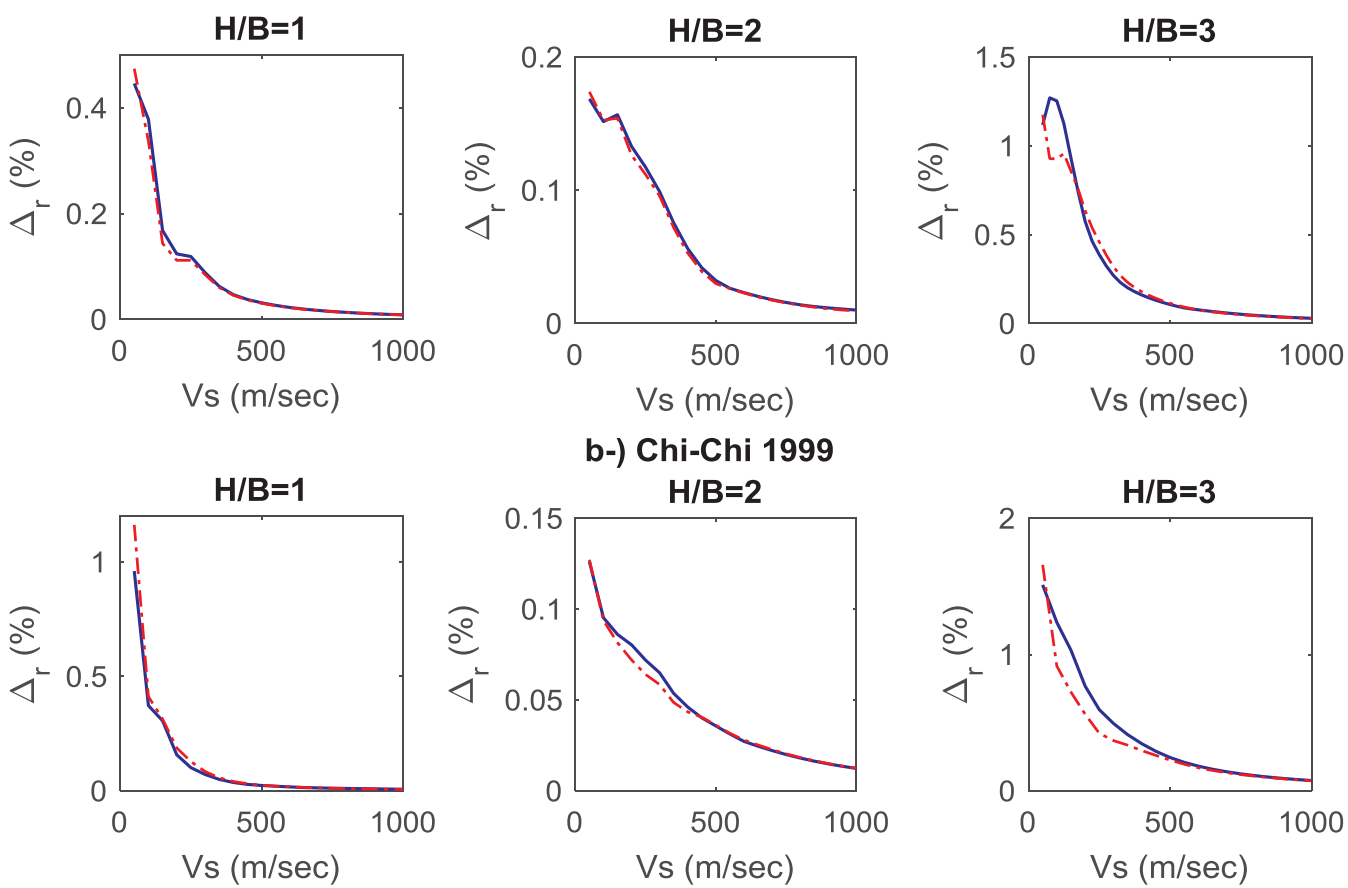

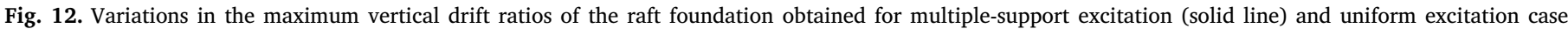
(dashed line). 
bending moments increase by $30 \%$. Similarly, for $\mathrm{H} / \mathrm{B}=3$, the maximum difference is in the range of $30 \%$ which is considered to partially reflect the change in the bending moment and shear forces. Since the change in these forces increases up to $60 \%$, the reason appears not to stem from only the additional drift due to time delay, but also vertical and lateral coupled modes seem to become effective. The maximum amplitude for $\mathrm{H} / \mathrm{B}=2$ is in the range of $0.15 \%$, and its difference from the uniform excitation is at most $10 \%$ in the vicinity of Vs $=250 \mathrm{~m} / \mathrm{sec}$.

\section{Conclusions}

In this study, the traveling wave effect of vertical ground motion in multi-story structures is investigated with soil structure interaction. The behavior of three numerical models with different heights $(\mathrm{H} / \mathrm{B}=1,2$, and 3) are investigated by considering two earthquake records with different characteristics. The identified results are summarized below.

- In the dynamic analysis, the effect of pseudo-static components on the higher modes become predominant as the soil stiffness increases. For soft soils, a few number of modes is adequate to induce all pseudo-static forces. The structural dynamic requirements on the modal contributions need to be considered separately.

- For slow shear wave velocities, the time delay causes a variation in the vertical drift ratio of the raft foundation. The first story column shear forces and bending moments show variations up to $60 \%$ due to this effect.

- The variation of the vertical drift may activate structural modes which is attributed to the very large changes in the columns axial forces. The numerical analysis shows that the axial forces at exterior columns can be as high as $200-250 \%$ of the results due to uniform excitation. For interior columns, the number increases up to $1200 \%$.

- According to the selected numerical models, as the height of the structure increases, the member forces are more affected by the time delay.

- Considering column design, axial load has a prominent effect on the columns flexural moment capacities. The observed change in this study, however, may cause a significant reduction in the flexural moment capacity of the designed columns in which a time delay effect is not considered.

\section{References}

[1] Soyluk K. Comparison of random vibration methods for multi-support seismic excitation analysis of long-span bridges. Eng Struct 2004;26(11):1573-83.

[2] Aviles J, Suarez M, Sanchez-Sesma FJ. Effects of wave passage on the relevant dynamic properties of structures with flexible foundation. Earthq Eng Struct D 2010;31:139-59.

[3] Zhang Z, Zhang Y, Lin J, Zhao Y, Howson WP, Williams FW. Random vibration of a train traversing a bridge subjected to traveling seismic waves. Eng Struct 2017;33:3546-58

[4] Jalali RS. A simplified multi-support response spectrum method. Earthq Eng Eng Vib 2012;11(2):243-56.

[5] Li J, Spencer Jr. VF, Elnashai AS, Philips BM. Substructure hybrid simulation with multiple-support excitation. J Eng Mech 2012;138(7):867-76.

[6] Ye J, Zhang Z, Liu X. A simplified multi-support response spectrum method. Earthq Eng Eng Vib 2012;11(2):243-56.

[7] Wang Z, Kiureghian AD. Multiple-support response spectrum analysis using loaddependent Ritz vectors. Earthq Eng Struct D 2014;43(15):2283-97.

[8] Mehanny SSF, Ramadan OMO, El Howary HA. Assessment of bridge vulnerability due to seismic excitations considering wave passage effects. Eng Struct 2014;70:197-207.

[9] Güllü H, Karabekmez B. Effect of near-fault and far-fault earthquakes on a historical masonry mosque through 3D dynamic soil-structure interaction. Eng Struct 2017;152:465-92.

[10] Cui PJ, Gao DF. Travelling wave effect analysis of long-span cable-stayed bridge. Adv Mat Res 2011;243-249:308-12.

[11] Jihong Y, Zhiqiang Z, Xianming L. A simplified multi-support response spectrum method. Earthq Eng Eng Vib 2011:11:243-56.

[12] Wang H, Li J, Tao TY, Wang C, Aiqun Li. Influence of apparent wave velocity on seismic performance of a super-long-span triple-tower suspension bridge. Adv Mech Eng 2015;7(6):1-14.

[13] Hizal C, Turan G. Travelling wave effect to the stress propagation in cable stayed bridges, 12nd Congress on Advances in Civil Engineering, Istanbul, Turkey, September; 2016.

[14] Rambabu KV, Allam MM. Response of an open-plane frame to multiple support horizontal seismic excitations with soil-structure interaction. J Sound Vib 2007;299(1-2):388-96.

[15] Allam MM. Multiple support excitations of open-plane frames by a filtered white noise and soil-structure interaction. J Sound Vib 2010;329(20):4212-26.

[16] Ghobarah A, Aziz TS, El-Attar M. Response of transmission lines to multiple support excitation. Eng Struct 1996;18(12):936-46.

[17] Tsai HC. Modal superposition method for dynamic analysis of structures excited by prescribed support displacements. Comput Struct 1998;66(5):675-83.

[18] Wilson EL. Three-dimensional Static and Dynamic Analysis of Structures. California, USA: Computers and Structures, Inc; 2002.

[19] CSI, SAP2000 Integrated software for structural analysis and design, Computers and Structures Inc, Berkeley, California.

[20] Mazzoni S, Mckenna F, Scott HM, Fenves GL. The open system for earthquake engineering simulation (OpenSEES) user command-language manual. Berkeley, C.A. University of California; 2006. [Pasific Earthquake Enineering Research Center].

[21] Hızal Ç, Turan G. Importance of static correction and damping in the analysis of a cable-stayed bridge subjected to displacement loading. J Bridge Eng 2017;22(6):04017009.

[22] Kramer SL. Geotechnical earthquake engineering. 1st ed. New Jersey: Prentice Hall; 1996. [USA].

[23] Chopra AK. Dynamics of structures theory and applications. 4th ed. New Jersey: Prentice Hall; 2013. [USA].

[24] Pais A, Kausel E. Approximate formulas for dynamic stiffnesses of rigid foundations. Soil Dyn Earthq Eng 1988;7(4):213-27.

[25] National Earthquake Hazards Reduction Program (NEHRP) Consultants Joint Venture. Soil structure interaction for building structures. National Institute of Standards and Technology, U.S. Department of Commerce; 2012. 